\title{
MicroRNA-361-5p suppresses cancer progression by targeting signal transducer and activator of transcription 6 in non-small cell lung cancer
}

\author{
YUEFENG MA ${ }^{1 *}$, CHUANEN BAO $^{2 *}$, RANRAN KONG $^{1}$, XIN XING $^{3}$, YAYA ZHANG $^{4}$, \\ SHAOMIN LI ${ }^{1}$, WEI ZHANG ${ }^{1}$, JIANTAO JIANG ${ }^{1}$, JIN ZHANG ${ }^{1}$, ZHE QIAO ${ }^{1}$, \\ DANJIE ZHANG ${ }^{1}$, ZHENCHUAN MA $^{1}$, LIANGZHANG SUN ${ }^{1}$ and BIN ZHOU ${ }^{1}$
}

\begin{abstract}
${ }^{1}$ Department of Thoracic Surgery, The Second Affiliated Hospital, Medical School of Xi'an Jiaotong University, Xi'an, Shaanxi 710004; ${ }^{2}$ Department of Thoracic and Cardiovascular Surgery,

The 174th Hospital of The Chinese People's Liberation Army, The Affiliated Chenggong Hospital of Xiamen University, Xiamen, Fujian 710049; ${ }^{3}$ Department of Health Care for Cadres, The Second Affiliated Hospital, Medical School of Xi'an Jiaotong University, Xi'an, Shaanxi 710004; ${ }^{4}$ Department of Oncology,

The 174th Hospital of The Chinese People's Liberation Army, The Affiliated Chenggong Hospital of Xiamen University, Xiamen, Fujian 710049, P.R. China
\end{abstract}

Received November 25, 2014; Accepted August 14, 2015

DOI: $10.3892 / \mathrm{mmr} .2015 .4411$

\begin{abstract}
The incidence of non-small cell lung cancer (NSCLC) has significantly increased in China, while the prognosis of affected patients is poor. The pathogenesis of NSCLC is thought to be regulated by microRNAs (miRs). The present study used a miR array in order to determine the expression of miR-361-5p, which was significantly lower in NSCLC tissues compared with that in adjacent tissues, indicating a crucial role of miR-361-5p during the progression of NSCLC. Furthermore, the effects of transfection-induced upregulation of miR-361-5p on the NSCLC cell line H23 were assessed. Overexpression of miR-361-5p inhibited the proliferation and colony formation ability of $\mathrm{H} 23$ cells. In addition, apoptosis of H23 cells was induced by upregulation of miR-361-5p. Furthermore, signal transducer and activator of transcription 6 (STAT6) was confirmed as a direct target of miR-361-5p by a dual-luciferase reporter assay. Moreover, inhibition of STAT6 by small interfering RNA or miR-361-5p also decreased the expression of $\mathrm{B}$-cell lymphoma extra large ( $\mathrm{Bcl}-\mathrm{xL})$. In vivo,
\end{abstract}

Correspondence to: Mr. Bin Zhou, Department of Thoracic Surgery, The Second Affiliated Hospital, Medical School of Xi'an Jiaotong University, 157 Xiwu Road, Xi'an, Shaanxi 710004, P.R. China

E-mail: zhoubin316@126.com

*Contributed equally

Key words: non-small cell lung cancer, microRNA-361-5p, signal transducer and activator of transcription 6, B-cell lymphoma extra large, progression
miR-361-5p significantly reduced tumor growth in a nude mouse xenograft model, and suppressed STAT6 and Bcl-xL expression. In conclusion, the present study indicated that miR-361-5p may represent a novel molecular tool for therapeutic and diagnostic strategies in NSCLC.

\section{Introduction}

The incidence of non-small cell lung cancer (NSCLC) has increased in recent years with the deterioration of air quality in China (1). In addition, mutations in oncogenes and tumor-suppressor genes trigger the progressive accumulation of NSCLC cases (2-5). Chinese patients with NSCLC have a high mortality rate and to date, limited therapeutic options are available for surgery-resistant NSCLC, leading to poor prognosis (1). Molecular targeting of cancer-associated signaling pathways represents a promising treatment option for cancer and has improved the 5-year survival rates of patients with metastatic cancer $(6,7)$. Although several targeted therapies, including the inhibition of BRAF (8), have been adopted for NSCLC treatment, challenges remain, similarly to those for other cancer types: i) Activation of collateral pathways often circumvents therapeutic blockage and induces resistance during targeted therapies; ii) Generic tumor-suppressive drugs are challenging to develop into clinical drugs.

MicroRNAs (miRNAs/miRs), small RNAs of 22 nucleotides in length, are non-coding single-stranded RNAs which are able to regulate gene expression via binding to the 3'-untranslated regions (UTR) of target mRNAs (9). Altered miRNA expression profiles have been reported in NSCLC, which result in differential expression of oncogenes and tumor suppressors, and affects downstream signaling pathways $(10,11)$. For instance, miRNA-21 (12), miRNA-205 (13), miR-1254 (14) and miR-574-5p (14) were found to be overexpressed in NSCLC. 
Furthermore, single-nucleotide polymorphisms in the complementary site of the 3'-UTR of target genes such as KRAS (rs712) are correlated with the risk of NSCLC, suggesting that let-7 miRs participate in the progression of NSCLC (15).

In the human genome, miR-361 is encoded by Xq21.2 between exons 9 and 10 of the choroideremia gene enconding for Rab escort protein 1 and produces two mature miRNAs, including miR-361-3p and miR-361-5p (16). miR-361-5p was downregulated in the cells of a transplantable metastatic xenograft compared to that in a non-metastatic xenograft (17). An in vitro study demonstrated that miR-361-5p was expressed in matched primary and metastatic carcinoma, which suggested that miR-361-5p inhibits the growth and proliferation of cancer cells (18). Kanitz et al (19) demonstrated that miR-361-5p is inversely correlated to vascular endothelial growth factor A in human cutaneous squamous cell carcinoma. In prostate cancer, miR-361-5p was shown to act as a tumor suppressor by targeting signal transducer and activator of transcription 6 (STAT6) (20). As it was hypothesized that miR-361-5p may also be a potential suppressor of other cancer types, the present study examined the effects of miR-361-5p in NSCLC.

The present study used miR-chips in order to determine the expression of miR-361-5p in NSCLC tissues and their adjacent normal tissues. Furthermore, the present study assessed the suppressive effects of miR-361-5p on the NSCLC cell line $\mathrm{H} 23$ using Cell Counting Kit 8 (CCK-8), colony formation and flow cytometric assays as well as a xenograft experiment. Transfection with miR-361-5p overexpression vector was used to upregulate miR-361-5p, and a luciferase reporter assay was used to identify a direct target as well as downstream signaling effects. Small-interfering (si)RNA was further used to knockdown a target gene of miR-361-5p in order to explore the downstream effects. The present study suggested that miR-361-5p inhibited NSCLC progression and may therefore be used as a novel therapeutic tool in NSCLC.

\section{Materials and methods}

Patients and samples. The NSCLC and adjacent tissues were obtained from 25 early-stage patients that underwent surgical resection and who had received no previous treatment. All patients were in T2 stage, including 7-patients with a Gleason score $<7,10$ patients with a Gleason score of 7 and 8 patients with a Gleason score $>7$ (21). All of these specimens were examined using haematoxylin and eosin (HE) staining (Beyotime Institute of Biotechnology, Inc., Wuhan, China). Only samples with $>70 \%$ tumor content were used as NSCLC tissues in the present study. The samples were frozen in liquid nitrogen for microarray and reverse-transcription polymerase chain reaction (RT-qPCR) analyses. All of the specimens were used according to institutional guidelines and approved protocols. The ethics committees of The Second Affiliated Hospital of the Medical School of Xi'an Jiaotong University (Xi'an, China) approved the protocol of the present study. Written informed consent was obtained from all patients.

Microarray analysis. The expression of miRNAs in NSCLC and adjacent tissues was assessed by miRNA-microarray. Briefly, total RNA was isolated using the mir-Vana ${ }^{\mathrm{TM}}$ miRNA isolation kit (Applied Biosystems, Thermo Fisher Scientific,
Waltham, MA, USA). After isolation of total RNA, individual RNA samples from the NSCLC and adjacent tissues were joined at equal amounts, respectively. One $\mu \mathrm{g}$ mixed RNA of NSCLC or adjacent tissues was reverse-transcribed into cDNA using a miRNA complete labeling and Hyb kit (Agilent Technologies, Inc., Santa Clara, CA, USA), respectively. Subsequently, the labeled probes were hybridized onto three miRNA microarrays (Agilent Technologies, Inc.) for each group. The Agilent SurePrint G3 Human GE 8x60K Microarrays were scanned and analyzed using Agilent Feature Extraction (v10.7) software (Agilent Technologies, Inc.). Agilent GeneSpring software 11.0 (Agilent Technologies, Inc.) was used for normalization of the expression levels using the $\Delta \Delta \mathrm{CT}$ method.

Cell culture. The H23 human NSCLC cell line was obtained from Shanghai Cell Bank, Chinese Academy of Sciences (Shanghai, China). The cells were cultured in Dulbecco's modified Eagle's medium (DMEM) (Gibco-BRL, Invitrogen Life Technologies, Inc., Carlsbad, CA, USA) with $10 \%$ fetal bovine serum (Gibco-BRL) at $37^{\circ} \mathrm{C}$ in an incubator with an atmosphere of $5 \% \mathrm{CO}_{2}$.

Cell transfection. The miRNA mimics and siRNA oligonucleotide duplexes were synthesised by GenePharma (Shanghai, China). The sequences of these miRNA mimics and siRNA oligonucleotide duplexes were those designed by a previous study (20): miR-361-5p, 5'-ACCCCUGGAGAUUCUGAUAA UU-3'; negative control (NC), 5'-UUCUCCGAACGUGUC ACGUTT-3'; STAT6-specific siRNA, 5'-AGACCUGUC CAUUCGCUCATT-3' (sense) and 5'-UGAGCGAAUGGA CAGGUCUTT-3' (anti-sense). NC, 5'-UUCUCCGAACGU GUCACGUTT-3' (sense) and 5'-ACGUGACACGUUCGG AGAATT-3' (anti-sense). Transfection was performed using Lipofectamine 2000 (Invitrogen Life Technologies) according to the manufacturer's instructions. The transfection complexes were added to the cells and incubated for $6 \mathrm{~h}$ prior to replacing the medium.

$R T-q P C R$. Total RNA was isolated from tissues or cells using RNAiso Plus (Takara, Dalian, China). The RNA concentration was measured using a BioPhotometer Plus (Eppendorf AG, Hamburg, Germany). RNA was reverse-transcribed into cDNA using a miScript SYBR Green PCR kit according to the manufacturer's instructions (Qiagen, Hilden, Germany). The primer for miR-361-5p was purchased from Sigma-Aldrich (St Louis, MO, USA; cat no. MIRAP00338). Reverse transcription was performed using a PrimeScript RT reagent kit with gDNA Eraser (Takara) according to the manufacturer's instructions. Applied Biosystems 7500 Real-Time PCR System was used to conduct the PCR. The PCR conditions were $95^{\circ} \mathrm{C}$ for 3 min followed by 40 cycles of $95^{\circ} \mathrm{C}$ for $12 \mathrm{sec}$ and $62^{\circ} \mathrm{C}$ for $40 \mathrm{sec}$. GAPDH was used as an internal control. The primers used were as follows: STAT6 forward, 5'-ACCCTCGAG TCCGCCACCATGGCTCTGTGGGGTCTG-3' and reverse, 5'-CAGCTGGGATCGAATTCTGGGGTTGGCCCT-3'; B-cell lymphoma extra large (Bcl-xL) forward, 5'-GATCCCCAT GGCAGCAGTAAAGCAAG-3' and reverse, 5'-CCCCATCCC GGAAGAGTTCATTCACT-3'. GAPDH forward, 5'-TGCACC ACCAACTGCTTAGC and reverse, 5'-GGCATGGACTGT GGTCATGAG-3'. All reactions were performed in triplicate. 
Negative control without template was used to eliminate contamination. The relative expression was calculated using the $2^{-\Delta \Delta \mathrm{Ct}}$ method.

Western blot analysis. The protein expression levels of STAT6, Bcl-xL and GAPDH were analyzed using western blot analysis. The cells were homogenized using NP-40 lysis buffer [1\% NP-40, $150 \mathrm{mM} \mathrm{NaCl}, 50 \mathrm{mM}$ Tris (pH 8.0)]. The protein concentration was determined using the bicinchoninic acid protein kit (Beyotime Institute of Biotechnology, Inc.). For each sample, $20 \mu \mathrm{g}$ protein was separated by $10 \%$ SDS-PAGE and transferred onto polyvinylidene fluoride membranes (Millipore, Billerica, MA, USA). After blocking with $4 \%$ skimmed milk at room temperature for $1 \mathrm{~h}$, the membranes were incubated with rabbit STAT6 monoclonal antibody (cat. no. \#5397; 1:1000; Cell Signaling Technology, Danvers, MA, USA), rabbit Bcl-xL monoclonal antibody (cat. no. sc-7195) or rabbit GAPDH monoclonal antibody (cat. no. sc-25778) (both 1:1000; Santa-Cruz Biotechnology, Inc., Dallas, TX, USA) at $4^{\circ} \mathrm{C}$ overnight, respectively. The membranes were then washed three times with TBST (pH 7.6; $20 \mathrm{mM}$ Tris- $\mathrm{HCl}$, $137 \mathrm{mM} \mathrm{NaCl}$ and $0.01 \%$ Tween-20). The membranes were subsequently stained with horseradish peroxidase-conjugated goat anti-rabbit secondary antibody (cat. no. sc-2301; 1:1000; Santa-Cruz Biotechnology, Inc.) at $37^{\circ} \mathrm{C}$ for $1 \mathrm{~h}$. The blots were visualized using enhanced chemiluminescence reagent (Millipore). Images were captured using a Kodak 440 digital imager (San Diego, CA, USA). Image-Pro Plus $\approx 6.0$ (Media Cybernetics, Inc., Rockville, MD, USA) was used for analysis.

Immunohistochemistry (IHC). The samples were fixed with $10 \%$ buffered formalin and embedded in paraffin. Tissue blocks were cut into $6-\mu \mathrm{m}$ sections. The sections were blocked with normal rabbit serum (Santa Cruz Biotechnology, Inc.)and then incubated with the same monoclonal antibodies as those used for western blotting (1:100 dilution for STAT6 and Bcl-xL) at $4^{\circ} \mathrm{C}$ overnight. After washing with TBST, the sections were stained with the horseradish peroxidase streptavidin complex (Beyotime Institute of Biotechnology, Inc.). The sections were color-developed with diaminobenzidine (Beyotime Institute of Biotechnology, Inc.). The sections were stained with hematoxylin and observed under a Leica 400 light microscope (Leica Microsystems, Inc., Buffalo Grove, IL, USA).

Cell proliferation assay. H23 cells were seeded in six-well plates and cultured for $24 \mathrm{~h}$ followed by transfection with miR-361-5p for $24 \mathrm{~h}$. Subsequently, the cells were re-suspended and seeded into a 96-well plate at 2,000 cells/well. Cell proliferation was determined using a CCK-8 assay kit (Beyotime Institute of Biotechnology, Inc). The absorbance was detected using a Bioluminescence 96-well microplate reader (GloMax 96-well Luminometer; Promega Corporation, Madison, WI, USA). at a wavelength of $450 \mathrm{~nm}$ according to the manufacturer's instructions. Cell viability was determined by calculating the mean values. Experiments were repeated three times and three wells were used for each group.

Colony formation assay. After transfection with miR-361-5p or NC vector, 300 cells for each group were plated into each well of a six-well plate and incubated for two weeks at $37^{\circ} \mathrm{C}$ in an incubator with an atmosphere of $5 \% \mathrm{CO}_{2}$. Subsequently, the cells were washed three times using phosphate-buffered saline (PBS) and fixed with methanol for $15 \mathrm{~min}$. The cells were then stained with $0.2 \%$ crystal violet (Beyotime Institute of Biotechnology, Inc.) for $20 \mathrm{~min}$ at room temperature. The colonies were counted and the results were obtained by determining the mean values from three independent experiments.

Flow cytometric analysis. To determine the apoptotic rate of the cells, Annexin V-fluorescein isothiocyanate and propidium iodide (BioVision, Milpitas, CA, USA) double staining was performed according to manufacturer's instructions, followed by flow cytometric analysis (BD FACSCanto II Flow; BeckmanCoulter, Brea, CA, USA). At least 30,000 cells for each sample were processed. The experiments were performed in triplicate.

Plasmid construction and luciferase reporter assay. The 3'-UTR of STAT6 mRNA with affinity for miR-361-5p was obtained by RT-PCR. The PCR products were then cloned into the psiCHECK-2 ${ }^{\mathrm{TM}}$ luciferase Vector (Promega Corporation) using XhoI and NotI restriction sites (Promega Corporation according to the protocol of a previous study (22). A vector containing a deletion at the binding site was also generated as a mutant reporter. The cells were then transfected with STAT6 3'-UTR (Sangon Biotech. Shanghai Co., Ltd., Shanghai, China) and mutant reporter as well as miR-361-5p mimics and NC. The Bcl-xL promoter region containing a STAT6-binding sequence was cloned into the luciferase reporter plasmid pGL3-basic (Promega Corporation) to generate a reporter of $\mathrm{Bcl}-\mathrm{xL}$. The cells were then transfected with these vectors and dual-luciferase assays were performed to detect luciferase activity at $48 \mathrm{~h}$ after co-transfection.

Xenograft experiment. Five-week-old female nude mice (age, 5 weeks; weight, $14.52 \pm 0.42 \mathrm{~g}$; $\mathrm{n}=5$ /group) were obtained from the Animal Center of The Second Affiliated Hospital (Medical School of Xi'an Jiaotong University, Xi'an, China) and were used for the tumor formation experiment. The mice were fed with a $0.012 \%$ rottlerin diet and kept at $22^{\circ} \mathrm{C}$ and $30 \%$ humidity. The experiment was approved by the ethics committee of the Second Affiliated Hospital of Medical School, Xi'an Jiaotong University (Xi'an, China). The mice were injected with H23 cells into the dorsal side. The tumor volume was measured every two days. The formula $\mathrm{V}=\mathrm{LxW} \mathrm{xDx} 3.14 / 6$ was used to calculate the tumor volume. Once the tumor volume was $>30 \mathrm{~mm}^{3}$, the mice were divided into two groups. One group was treated with 200 pmol NC vector and the other group was treated with 200 pmol miR-361-5p mimics with $10 \mu 1$ Lipofectamine 2000 (Invitrogen Life Technologies, Inc.). The xenograft tumors were injected with the vectors at multiple sites and injection was performed every 2 days over 19 days. The tumors were harvested following 19 days and subjected to western blot analysis, IHC and RT-qPCR. After 19 days post-experiment, the mice were anesthetized by inhalation of $2.5 \%$ isoflurane (Invitrogen Life Technologies, Inc.) and executed.

Statistical analysis. Statistical analysis was performed using SPSS 17.0 (SPSS Inc., Chicago, IL, USA). Values are expressed as the mean \pm standard deviation of at least three independent 
A
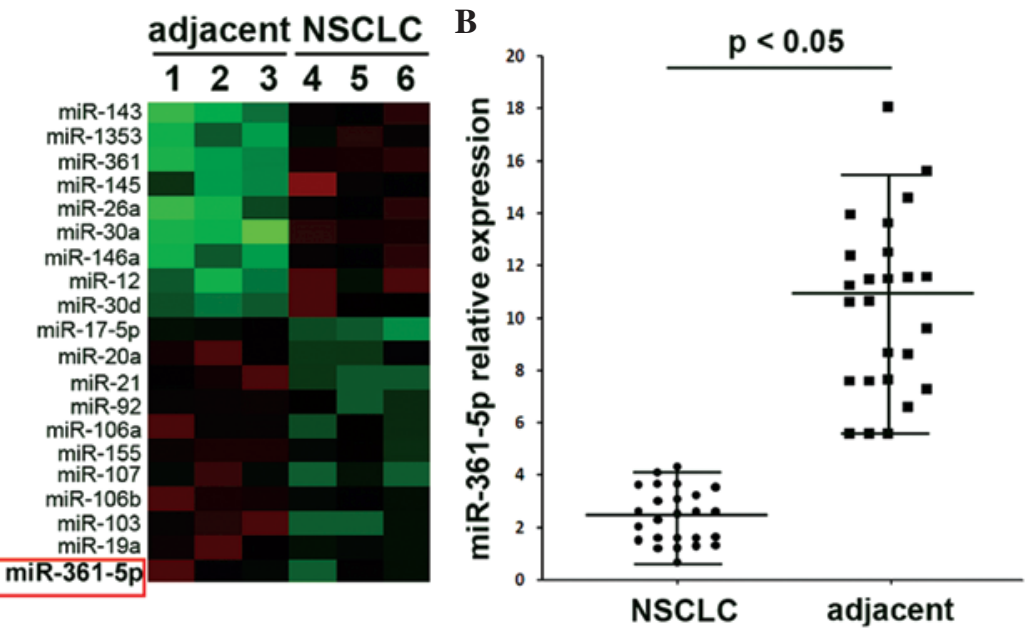

Figure 1. Reduced expression of miR-361-5p in NSCLC. (A) Heat map of the expression profiles of miRs in three NSCLC tissues and three adjacent tissues which was analyzed by Agilent microarrays. (B) Reverse-transcription polymerase chain reaction results indicate lower expression of miR-361-5p in NSCLC tissues than that in adjacent tissues. Each data-point represents the result for one tumor. Horizontal lines represent the mean value and bars represent the standard deviation. miR, microRNA; NSCLC, non-small cell lung cancer.

A

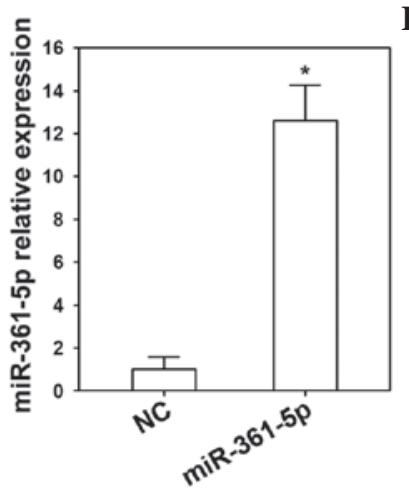

D

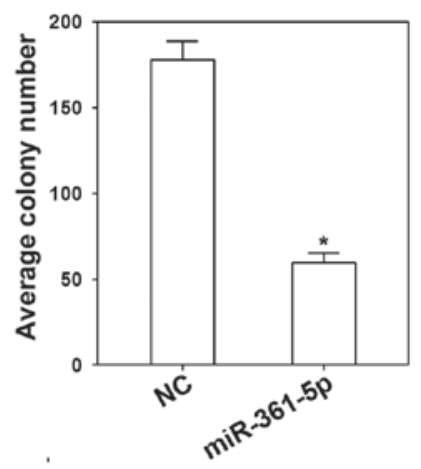

B

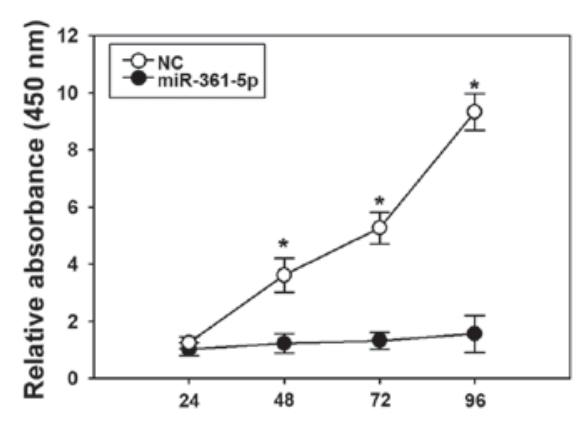

$\mathbf{E}$

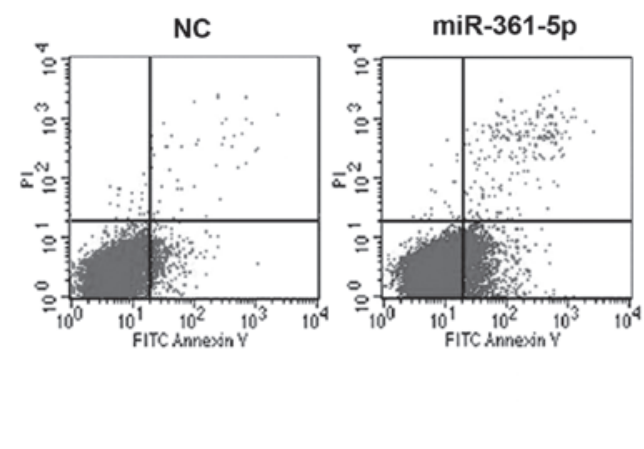

C

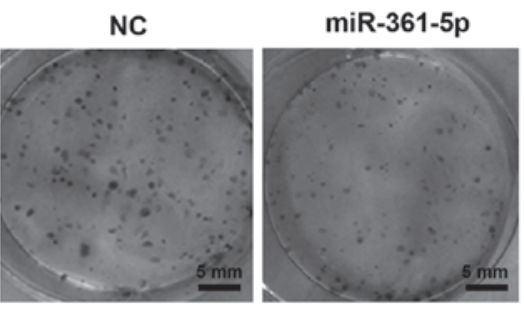

F

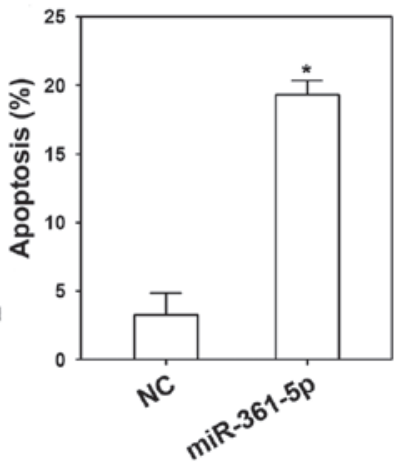

Figure 2. Effect of miR-361-5p on H23 cells. (A) miR-361-5p transfection increased the expression of miR-361-5p in H23 cells. (B) A Cell Counting Kit 8 assay indicated that overexpression of miR-361-5p reduced H23 cell proliferation. (C and D) Overexpression of miR-361-5p reduced the colony-forming efficiency of $\mathrm{H} 23$ cells. (E and F) Overexpression of miR-361-5p increased apoptosis of $\mathrm{H} 23$ cells as indicated by flow cytometric analysis. Values are expressed as the mean \pm standard deviation. " $\mathrm{P}<0.05$ vs. NC group. NC, negative control; miR, microRNA; FITC, fluorescein isothiocyanate; PI, propidium iodide.

experiments. Significant differences were confirmed using one-way analysis of variance. $\mathrm{P}<0.05$ was considered to indicate a statistically significant difference.

\section{Results}

miR-361-5p is downregulated in NSCLC tissues. The miRNA array of NSCLC samples and adjacent tissues indicated lower expression of miR-361-5p in NSCLC tissues compared to that in adjacent tissues (Fig. 1A). Expressional changes of miR-361-5p in 25 NSCLC and 25 adjacent tissues were also confirmed by RT-qPCR (Fig. 1B) with the results showing similar results to those of the miRNA array. It is therefore concluded that the reduced expression of miR-361-5p may participate in the progression of NSCLC.

Effects of miR-361-5p on H23 in vitro. The effects of miR-361-5p in H23 cells were detected by transfection with 
A

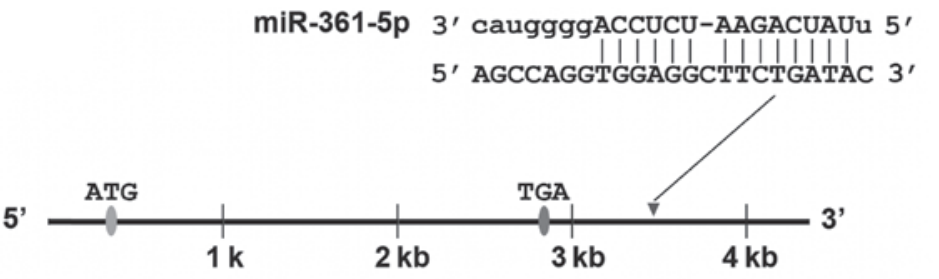

C

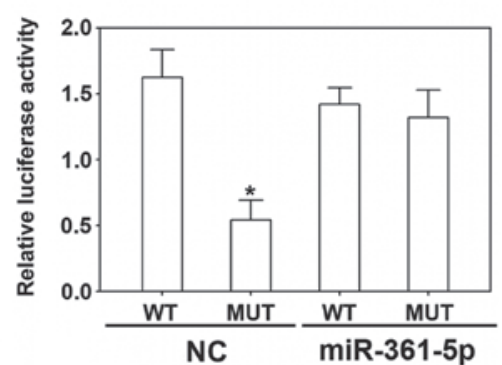

B

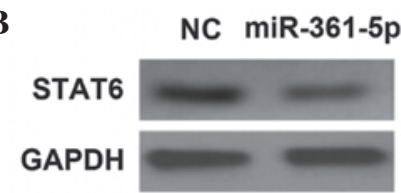

$\mathbf{E}$

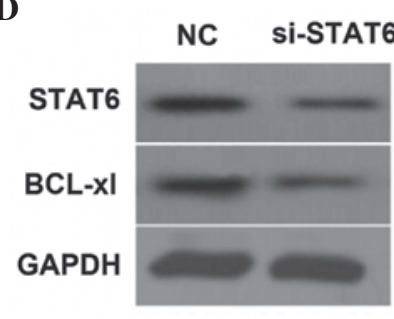

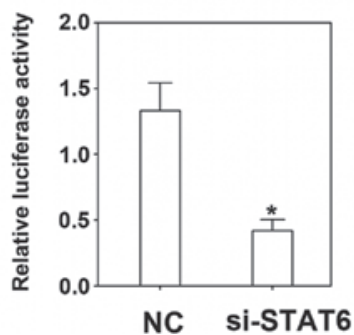

Figure 3. miR-361-5p regulates the expression of STAT6 and BCL-xl. (A) miR-361-5p targets the 3'-UTR of STAT6. (B) STAT6 protein levels were reduced following overexpression of miR-361-5p. (C) A dual luciferase reporter assay using the psiCHECK-2 vector containing the STAT6 3'-UTR showed that luciferase activity was decreased following upregulation of miR-361-5p expression. ${ }^{*} \mathrm{P}<0.05$ vs. WT. (D) STAT6 and Bcl-xL protein levels were reduced following treatment with si-STAT6. (E) Luciferase reporter assay showing that si-STAT6 suppressed the Bcl-xL promoter activity. Values are expressed as the mean \pm standard deviation. "P<0.05 vs NC. STAT6, signal transducer and activator of transcription 6; Bcl-xL, B-cell lymphoma extra large; WT, wild-type; MUT, mutant; NC, negative control; miR, microRNA; si-STAT6, small interfering RNA targeting STAT6; 3'-UTR, 3'-untranslated region.

A

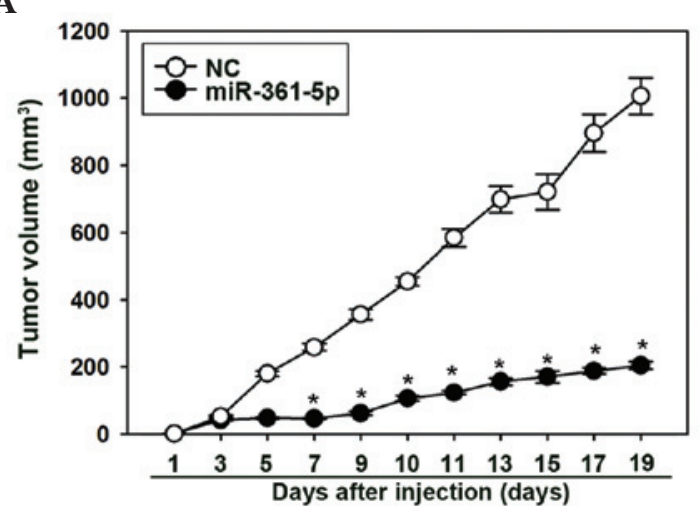

C

NC miR-361-5p
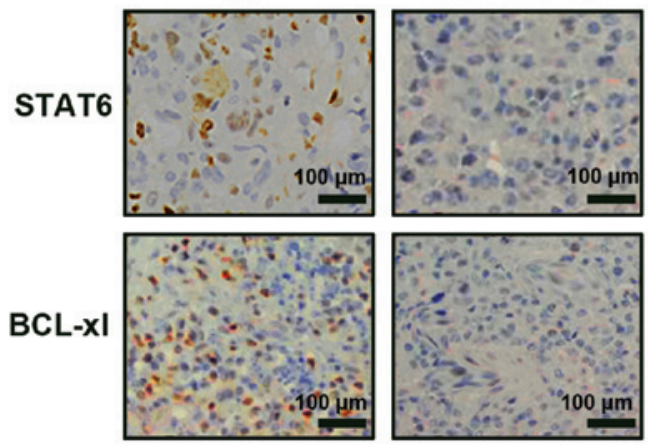

B
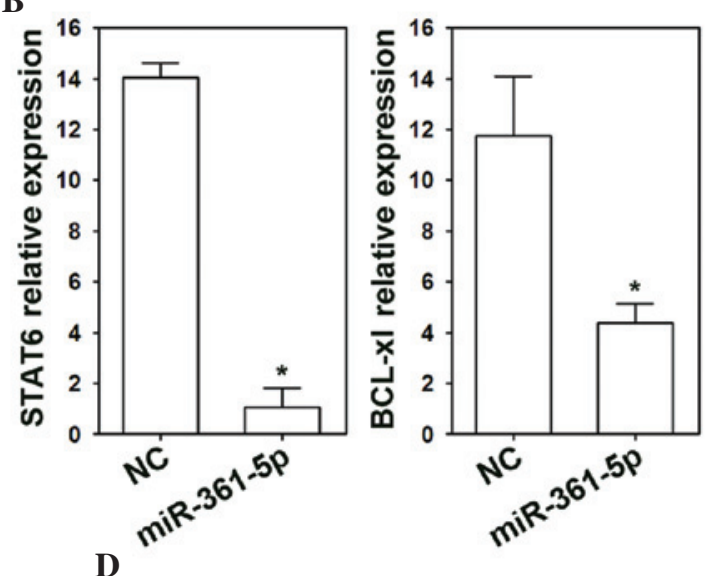

NC miR-361-5p

STAT6

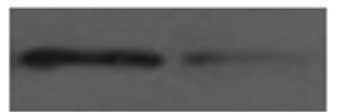

BCL-XI

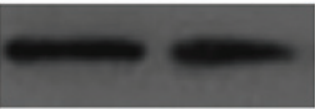

GAPDH

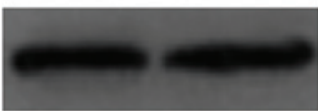

Figure 4. miR-361-5p reduces tumor growth as well as the expression of STAT6 and BCL-xL in xenografts grown in nude mice. (A) miR-361-5p injection markedly inhibited tumor growth in a nude mouse xenograft model. (B) Reverse-transcription polymerase chain reaction analysis suggested that overexpression of miR-361-5p markedly reduced the mRNA expression of STAT6 and BCL-xL. Values are expressed as the mean \pm standard deviation. ${ }^{*} \mathrm{P}<0.05 \mathrm{vs}$. NC (C and D) Immunohistochemical and western blot analyses indicated depressive effects of miR-361-5p on the protein expression levels of STAT6 and BCL-xL in xenograft tumor tissues. Figures are representative of three separate experiments. STAT6, signal transducer and activator of transcription 6; Bcl-xL, B-cell lymphoma extra large; NC, negative control; miR, microRNA.

miR-361-5p mimics or NC vector. RT-qPCR confirmed that miR-361-5p was overexpressed in the miR-361-5p mimics group compared to that in the NC group (Fig. 2A). Of note, overexpression of miR-361-5p inhibited the proliferation and colony formation ability of $\mathrm{H} 23$ cells (Fig. 2B-D). Flow cytometric analysis showed that the apoptotic rate of $\mathrm{H} 23$ cells 
transfected with miR-361-5p mimics was higher than that in the NC group (Fig. 2E and F).

STAT6 is a direct target of miR-361-5p and promotes Bcl-xL expression. The 3'-UTR of STAT6 mRNA was identified as a target of miR-361-5p (Fig. 3A). In the present study, the mRNA and protein levels of STAT6 were depressed by transfection with miR-361-5p mimics (Fig. 3B and C). To confirm the direct interaction of miR-361-5p with the STAT6 3'-UTR, a dual-luciferase reporter vector containing the STAT6 3'-UTR was constructed and transfected into $\mathrm{H} 23$ cells, which were co-transfected with miR-361-5p mimics or control vector. The results showed that luciferase activity was significantly decreased when miR-361-5p was overexpressed. Moreover, following transfection with STAT6-specific siRNA, the expression of Bcl-xL was downregulated, indicating that STAT6 is required for Bcl-xL expression in $\mathrm{H} 23$ cells (Fig. 3D). A further luciferase reporter assay demonstrated that STAT6 was able to bind to the Bcl-xL promoter and that STAT6 siRNA was able to decrease Bcl-xL expression and luciferase activity (Fig. 3E).

miR-361-5p suppresses tumor growth in vivo. Following transfection with miR-361-5p every other day, the volume of tumors derived from $\mathrm{H} 23$ cells was significantly suppressed from days 5-18 of the xenograft experiment (Fig. 4A). Vector-mediated overexpression of miR-361-5p in the tumors led to the downregulation of STAT6 and Bcl-xL at the mRNA as well as the protein level (Fig. 4B-D), which indicated that miR-361-5p reduced STAT6/Bcl-xL signaling and tumorigenicity in vivo.

\section{Discussion}

NSCLC is one of the most prevalent cancer types in China and has a high mortality rate; however, the underlying mechanisms for the progression of NSCLC have largely remained elusive. Accumulating evidence has indicated a deregulated expression of miRNAs in various types of cancer, including NSCLC $(11,23,24)$. These miRNAs are likely to have crucial roles in tumorigenesis and may be employed as suitable targets or tools in cancer treatment. For instance, miR-21 represses tumor suppressor phosphatase and tensin homolog and promotes growth (25) and invasion of NSCLC, while miR-494 is able to modulate apoptosis induced by tumor necrosis factor-related apoptosis-inducing ligand in NSCLC (26). In addition, miR-101, miR-1254, miR-574-5p, miR-143 and miR-181a were demonstrated to be involved in $\operatorname{NSCLC}(27,28)$. The present study demonstrated that miR-361-5p was downregulated in NSCLC tissues compared with that in adjacent tissues, which suggested that miR-361-5p may inhibit the progression of NSCLC. Thus, the present study further examined the role of miR-361-5p in the proliferation of proliferation NSCLC cells in vitro as well as in vivo.

The present study demonstrated that transfection of H23 cells with miR-361-5p suppressed their proliferation and colony formation ability. Furthermore, overexpression of miR-361-5p induced apoptosis in H23 cells. These results indicated that miR-361-5p acted as a tumor suppressor in NSCLC. Similar results were previously obtained for the castration-resistant prostate cancer cell line DU145 (20). By contrast, miR-361-5p functioned as an oncogene in cervical carcinoma cells by enhancing their proliferation; it was indicated that miR-361-5p is an important factor for the progression of cervical cancer (29). Due to these contradictory results, further studies should scan different types of cancer in order to elucidate the function of miR-361-5p.

STAT6, a member of the STAT family, is able to activate cytokines and growth factors (30). In cancers, the expression of STAT6 was shown to be upregulated $(31,32)$. Cui et al $(33)$ found that unphosphorylated STAT6 triggers the upregulation of cyclooxygenase- 2 in NSCL. In addition, they demonstrated that knockout of STAT6 resulted in a decrease of cyclooxygenase- 2 expression. Cyclooxygenase- 2 is a well-known marker of malignant cancer phenotypes (34). Thus, STAT6 also participates in the proliferation and invasion of cancer cells. In mammary carcinoma, STAT6-deficient mice showed a markedly enhanced immunity against tumors and metastasis (35). In prostate cancer, miR-361-5p inhibited STAT6 as its target gene, and overexpression of miR-361-5p in prostate neoplasms depressed STAT6 in vivo as well as in vitro (20). In analogy with this, the results of the present study also showed that in NSCLC, miR-361-5p inhibited the expression of STAT6 in vivo as well as in vitro. In order to further elucidate the roles of the STAT6/Bcl-xL signaling pathway, the present study examined the expression of Bcl-xL following STAT6 inhibition and used a luciferase reporter assay to demonstrate that STAT6 directly regulates Bcl-xL expression in NSCLC.

The crosstalk between the STAT6/Bcl-xL signaling pathway and miR-361-5p may be a key incidence for the development of metastatic of NSCLC. STAT6 knockout was previously associated with the depression of proliferation and induction of apoptosis of cancer cells (33). Furthermore, STAT6 signaling was shown to contribute to cancer cell growth. The present study demonstrated that Bcl-xL levels were reduced following downregulation of STAT6 by miR-361-5p, providing a novel approach for treating NSCLC by inhibiting the STAT6/Bcl-xL signaling pathway. Several miRNAs have been previously shown to inhibit the proliferation and metastasis of tumor cells. However, to date, no suitable therapeutic targets for NSCLC have been identified. In order to uncover therapeutic targets, a comprehensive screening analysis of miRNAs in NSCLC is required. The present study used a miRNA array to scan NSCLC tissues for potential targets, revealing a regulatory mechanism of miR-361-5p in NSCLC. It was speculated that the downregulation of miR-361-5p may be responsible for the dysregulation of STAT6/Bcl-xL signaling, resulting in tumor progression.

In conclusion, the present study demonstrated that miR-361-5p was decreased in NSCLC tissues. Furthermore, upregulation of miR-361-5p depressed proliferation and colony formation, and promoted apoptosis of $\mathrm{H} 23$ cells via the STAT6/Bcl-xL pathway in vitro and in vivo. These results indicated that $\mathrm{miR}-361-5 \mathrm{p}$ has a tumor-suppressive function in NSCLC, which may be harnessed as a strategy for treating NSCLC; the feasibility of this approach requires further evaluation.

\section{Acknowledgements}

The present study was supported in part by a grant from the Science and Technology R\&D Program of Shaanxi Province [Grant no. 2010K14-02 (22)]. 


\section{References}

1. Xue C, Hu Z, Jiang W, Zhao Y, Xu F, Huang Y, Zhao H, Wu J, Zhang Y, Zhao L, et al: National survey of the medical treatment status for non-small cell lung cancer (NSCLC) in China. Lung Cancer 77: 371-375, 2012.

2. Paez JG, Jänne PA, Lee JC, Tracy S, Greulich H, Gabriel S, Herman P, Kaye FJ, Lindeman N, Boggon TJ, et al: EGFR mutations in lung cancer: Correlation with clinical response to gefitinib therapy. Science 304: 1497-1500, 2004

3. Kosaka T, Yatabe Y, Endoh H, Kuwano H, Takahashi T and Mitsudomi T: Mutations of the epidermal growth factor receptor gene in lung cancer: Biological and clinical implications. Cancer Res 64: 8919-8923, 2004

4. Mitsudomi T, Viallet J, Mulshine JL, Linnoila RI, Minna JD and Gazdar AF: Mutations of ras genes distinguish a subset of non-small-cell lung cancer cell lines from small-cell lung cancer cell lines. Oncogene 6: 1353-1362, 1991

5. Stephens P, Hunter C, Bignell G, Edkins S, Davies H, Teague J, Stevens C, O'Meara S, Smith R, Parker A, et al: Lung cancer: Intragenic ERBB2 kinase mutations in tumours. Nature 431: 525-526, 2004

6. Temel JS, Greer JA, Muzikansky A, Gallagher ER, Admane S, Jackson VA, Dahlin CM, Blinderman CD, Jacobsen J, Pirl WF, et al: Early palliative care for patients with metastatic non-small-cell lung cancer. N Engl J Med 363: 733-742, 2010.

7. Schilsky RL: Personalized medicine in oncology: The future is now. Nat Rev Drug Discov 9: 363-366, 2010.

8. Gerber DE and Minna JD: ALK inhibition for non-small cell lung cancer: From discovery to therapy in record time. Cancer Cell 18: 548-551, 2010.

9. McManus MT and Sharp PA: Gene silencing in mammals by small interfering RNAs. Nat Rev Genet 3: 737-747, 2002.

10. Yu SL, Chen HY, Chang GC, Chen CY, Chen HW, Singh S, Cheng CL, Yu CJ, Lee YC, Chen HS, et al: MicroRNA signature predicts survival and relapse in lung cancer. Cancer Cell 13: 48-57, 2008

11. Yanaihara N, Caplen N, Bowman E, Seike M, Kumamoto K, Yi M, Stephens RM, Okamoto A, Yokota J, Tanaka T, et al: Unique microRNA molecular profiles in lung cancer diagnosis and prognosis. Cancer Cell 9: 189-198, 2006.

12. Wang ZX, Bian HB, Wang JR, Cheng ZX, Wang KM and De W: Prognostic significance of serum miRNA-21 expression in human non-small cell lung cancer. J Surg Oncol 104: 847-851, 2011.

13. Larzabal L, de Aberasturi AL, Redrado M, Rueda P, Rodriguez MJ, Bodegas ME, Montuenga LM and Calvo A: TMPRSS4 regulates levels of integrin $\alpha 5$ in NSCLC through miR-205 activity to promote metastasis. Br J Cancer 110: 764-774, 2014.

14. Foss KM, Sima C, Ugolini D, Neri M, Allen KE and Weiss GJ: MiR-1254 and miR-574-5p: Serum-based microRNA biomarkers for early-stage non-small cell lung cancer. J Thorac Oncol 6 : 482-488, 2011.

15. Chin LJ, Ratner E, Leng S, Zhai R, Nallur S, Babar I, Muller RU, Straka E, Su L, Burki EA, et al: A SNP in a let-7 microRNA complementary site in the KRAS 3' untranslated region increases non-small cell lung cancer risk. Cancer Res 68: 8535-8540, 2008.

16. Roth C, Stückrath I, Pantel K, Izbicki JR, Tachezy M and Schwarzenbach H: Low levels of cell-free circulating miR-361-3p and miR- $625^{*}$ as blood-based markers for discriminating malignant from benign lung tumors. PLoS One 7: e38248, 2012.

17. Watahiki A, Wang Y, Morris J, Dennis K, O'Dwyer HM, Gleave M, Gout PW and Wang Y: MicroRNAs associated with metastatic prostate cancer. PLoS One 6: e24950, 2011

18. Khella HW, White NM, Faragalla H, Gabril M, Boazak M, Dorian D, Khalil B, Antonios H, Bao TT, Pasic MD, et al: Exploring the role of miRNAs in renal cell carcinoma progression and metastasis through bioinformatic and experimental analyses. Tumor Biol 33: 131-140, 2012.
19. Kanitz A, Imig J, Dziunycz PJ, Primorac A, Galgano A Hofbauer GF, Gerber AP and Detmar M: The expression levels of microRNA-361-5p and its target VEGFA are inversely correlated in human cutaneous squamous cell carcinoma. PLoS One 7: e49568, 2012.

20. Liu D, Tao T, Xu B, Chen S, Liu C, Zhang L, Lu K, Huang Y, Jiang L, Zhang X, et al: MiR-361-5p acts as a tumor suppressor in prostate cancer by targeting signal transducer and activator of transcription-6 (STAT6). Biochem Biophys Res Commun 445: 151-156, 2014.

21. Humphrey PA: Gleason grading and prognostic factors in carcinoma of the prostate. Mod Pathol 17: 292-306, 2004.

22. Kertesz M, Iovino N, Unnerstall U, Gaul U and Segal E: The role of site accessibility in microRNA target recognition. Nature Genetics 39: 1278-1284, 2007.

23. Rabinowits G, Gerçel-Taylor C, Day JM, Taylor DD and Kloecker GH: Exosomal microRNA: A diagnostic marker for lung cancer. Clin Lung Cancer 10: 42-46, 2009.

24. Lu J, Getz G, Miska EA, Alvarez-Saavedra E, Lamb J, Peck D, Sweet-Cordero A, Ebert BL, Mak RH, Ferrando AA, et al: MicroRNA expression profiles classify human cancers. Nature 435: 834-838, 2005.

25. Zhang JG, Wang JJ, Zhao F, Liu Q, Jiang $\mathrm{K}$ and Yang GH MicroRNA-21 (miR-21) represses tumor suppressor PTEN and promotes growth and invasion in non-small cell lung cancer (NSCLC). Clin Chim Acta 411: 846-852, 2010.

26. Romano G, Acunzo M, Garofalo M, Di Leva G, Cascione L, Zanca C, Bolon B, Condorelli G and Croce CM: MiR-494 is regulated by ERK1/2 and modulates TRAIL-induced apoptosis in non-small-cell lung cancer through BIM down-regulation. Proc Natl Acad Sci USA 109: 16570-16575, 2012.

27. Guan P, Yin Z, Li X, Wu W and Zhou B: Meta-analysis of human lung cancer microRNA expression profiling studies comparing cancer tissues with normal tissues. J Exp Clin Cancer Res 31: 54, 2012.

28. Wang Q, Wang S, Wang H, Li P and Ma Z: MicroRNAs: Novel biomarkers for lung cancer diagnosis, prediction and treatment. Exp Biol Med (Maywood) 237: 227-235, 2012.

29. Wu X, Xi X, Yan Q, Zhang Z, Cai B, Lu W and Wan X: MicroRNA-361-5p facilitates cervical cancer progression through mediation of epithelial-to-mesenchymal transition. Med Oncol 30: 751, 2013.

30. Wurster AL, Tanaka T and Grusby MJ: The biology of Stat 4 and Stat6. Oncogene 19: 2577-2584, 2000.

31. Das S, Shetty P, Valapala M, Dasgupta S, Gryczynski Z and Vishwanatha JK: Signal transducer and activator of transcription 6 (STAT6) is a novel interactor of annexin A2 in prostate cancer cells. Biochemistry 49: 2216-2226, 2010.

32. Li BH, Yang XZ, Li PD, Yuan Q, Liu XH, Yuan J and Zhang WJ: IL-4/Stat6 activities correlate with apoptosis and metastasis in colon cancer cells. Biochem Biophys Res Commun 369: 554-560, 2008.

33. Cui X, Zhang L, Luo J, Rajasekaran A, Hazra S, Cacalano N and Dubinett SM: Unphosphorylated STAT6 contributes to constitutive cyclooxygenase-2 expression in human non-small cell lung cancer. Oncogene 26: 4253-4260, 2007.

34. Hida T, Yatabe Y, Achiwa H, Muramatsu H, Kozaki K, Nakamura S, Ogawa M, Mitsudomi T, Sugiura T and Takahashi T: Increased expression of cyclooxygenase 2 occurs frequently in human lung cancers, specifically in adenocarcinomas. Cancer Res 58: 3761-3764, 1998.

35. Ostrand-Rosenberg S, Grusby MJ and Clements VK: Cutting edge: STAT6-deficient mice have enhanced tumor immunity to primary and metastatic mammary carcinoma. J Immunol 165: 6015-6019, 2000 